\title{
PALEOAMBIENTE DE LA SECUENCIA MAÍZ GORDO (¿PALEOCENO TARDÍO-EOCENO TEMPRANO?), ARROYO LAS TORTUGAS, CUENCA DEL GRUPO SALTA (NO ARGENTINA). PALINOLOGÍA Y SEDIMENTOLOGÍA
}

\author{
Mirta QUATTROCCHIO' y Cecilia DEL PAPA ${ }^{2}$ \\ ' Departamento de Geología. Universidad Nacional del Sur. San Juan 670. (8000) Bahía Blanca. \\ CONICET. \\ 2 Facultad de Ciencias Naturales. Universidad Nacional de Salta. Buenos Aires 177. (4400) Salta. \\ CONICET.
}

Quattrocchio, M. y Del Papa, C. 2000. Paleoambiente de la Secuencia Maíz Gordo (¿Paleoceno Tardío-Eoceno Temprano?), arroyo Las Tortugas, Cuenca del Grupo Salta (NO Argentina). Palinología y sedimentología. [Palaeoenvironment of the Maíz Gordo Sequence (Late Paleocene-Early Eocene?), Las Tortugas stream, Salta Group Basin (NW Argentina). Palynology and sedimentology]. Revista Española de Paleontología, 15(1), 57-70. ISSN 0213-6937.

\begin{abstract}
Four sedimentary sub-environments have been recognized in the Maíz Gordo Sequence exposed in the Las Tortugas stream Salta Group Basin. They were named: mud plain, carbonate plain, stratificated lacustrine, and lacustrine wave-dominated depositional systems. The carbonate plain and stratificated lacustrine depositional systems were analyzed taking into account the sedimentary facies and palynofacies. Opaque phytoclasts dominate the laminated dolomicritic mudstone facies and green claystone and siltstone facies. Amorphous organic matter dominates the laminated limestone facies associated with dinoflagellate non-marine cysts and gypsum. A new non-marine dinoflagellate cyst is recognized. A lacustrine environment with alternating periods of flooding and shallowing is inferred for Maíz Gordo Sequence (Middle Section) in Las Tortugas stream locality.
\end{abstract}

Keywords: Palaeoenvironment, palynology, sedimentology, Paleocene-Lower Eocene?, Maíz Gordo Sequence, Salta Group, Argentina.

\section{RESUMEN}

Cuatro subambientes sedimentarios han sido reconocidos en la Secuencia Maíz Gordo expuesta en el arroyo Las Tortugas, Cuenca del Grupo Salta. Ellos fueron denominados: llanura de fango, llanura carbonática, lacustre estratificado y lacustre dominado por barras de oleaje. Los sistemas deposicionales de llanura carbonática y lacustre estratificado fueron analizados teniendo en cuenta las facies sedimentarias y las palinofacies. Los fitoclastos opacos dominan la facies de mudstone dolomicrítico laminado y la facies de arcilita y limolitas verdes. La materia orgánica amorfa domina en la facies de calizas laminadas, asociada con quistes de dinoflagelados no-marinos. Se reconoce un nuevo quiste de dinoflagelado no-marino. La Secuencia Maíz Gordo (Sección Media) en la localidad de Las Tortugas está caracterizada por sedimentación pelítica-carbonática ocurrida en una cuenca lacustre con períodos alternantes de inundación-somerización.

Palabras clave: Paleoambiente, palinología, sedimentología, ¿Paleoceno-Eoceno temprano?, Secuencia Maíz Gordo, Grupo Salta, Argentina.

\section{INTRODUCCIÓN}

El presente trabajo tiene como finalidad evaluar el paleoambiente de sedimentación de la Formación Maíz Gordo (Subgrupo Santa Bárbara, Grupo Salta) en el arroyo Las Tortugas. Para tal fin se utilizaron como herramientas el análisis de facies y secuencias de facies, así como el estudio palinológico. Este último comprende el análisis tradicional de asignación de los taxa fósiles a los taxa modernos relacionados, evaluando de esta 


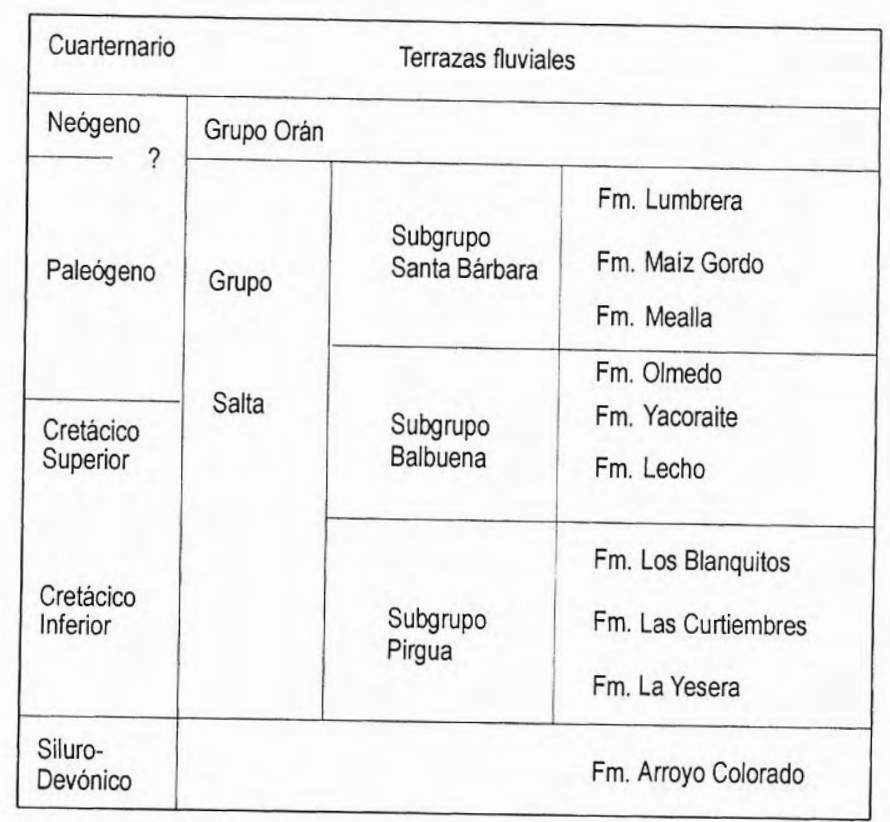

Figura 1. Cuadro estratigráfico del Grupo Salta.

manera las paleocomunidades vegetales existentes y además, el análisis palinofacial, que comprende el estudio total de la materia palinológica (palinofacies) presente en un preparado palinológico.

La interpretación de facies se basa sólamente en lo que ha sido conservado. Los sedimentos son comúnmente removidos por erosión y la mayoría de los restos orgánicos son destruidos, por lo que la interpretación de las facies y palinofacies es sólo una aproximación del paleoambiente real de sedimentación.

Se realiza, además, una evaluación palinoestratigráfica de la Formación Maíz Gordo basada en el rango estratigráfico de los palinomorfos hallados y se incluye una comparación con registros previos de la mencionada formación (Volkheimer et al., 1984) y con el resto de las unidades que integran el Subgrupo Santa Bárbara del Grupo Salta (Quattrocchio 1978 a, b y 1980; Quattrocchio y Volkheimer 1988, 1990 y en prensa; Quattrochio et al. 1988 y 1997).

Se incluye la lista sistemática de las especies halladas y la descripción sistemática de una nueva especie de quiste de dinoflagelado no-marino.

\section{ESTRATIGRAFÍA}

La Formación Maíz Gordo (Moreno, 1970) integra, junto con las formaciones Mealla y Lumbrera, el Subgrupo Santa Bárbara, Grupo Salta (Turner, 1959). Estos depósitos representan el final del ciclo estratigráfico del Cretácico-Eoceno en el noroeste argentino (Salfity, 1982) (Fig. 1).

El Grupo Salta se formó en una cuenca de rift intracontinental, durante el Cretácico Inferior-Paleógeno (Salfity, 1980, Galliski y Viramonte, 1988). Los depósitos sinrift están representados por el Subgrupo Pirgua (Kimmeridgiense?- pre-Maastrichtiense), mientras que los depósitos postrift están integrados por los subgrupos Balbuena y Santa Bárbara (Maastrichtiense Eoceno), que colmataron la cuenca.

Recientemente la Formación Maíz Gordo ha sido definida con el rango de Secuencia Maíz Gordo (Del Papa, 1992), estableciéndose los limites discordantes con las secuencias Mealla y Lumbrera, según los criterios de la estratigrafía secuencial (Vail et al., 1977).

La Secuencia Maíz Gordo se depositó en una cuenca prácticamente nivelada, con escaso relieve circundante. Como consecuencia, la velocidad de subsidencia era baja, lo cual asociado a una baja tasa de sedimentación y un marcado ascenso del nivel de base generaron una tendencia retrogradante de la secuencia. Está integrada por sedimentitas depositadas en una cuenca lacustre, somera, de aguas alcalinas y salinas; asociada lateralmente a sistemas fluviales de tipo entrelazado arenoso y abanicos aluviales arenosos, localizados próximos a las áreas de aporte (Gómez Omil et al., 1989, Del Papa, 1994).

El material recolectado en el presente estudio proviene de la localidad de Las Tortugas, ubicada al norte de la sierra de Maíz Gordo (Sierras Subandinas) (Fig. 2). Estos afloramientos, de 200 a $250 \mathrm{~m}$ de espesor, representan parte de la sedimentación ocurrida en la subcuenca de Lomas de Olmedo, cuyo mayor registro sedimentario actualmente se encuentra en subsuelo. Esta subcuenca presentó para el tiempo de depósito del Grupo Salta una alta tasa de subsidencia que provocó la acumulación de una potente y prácticamente completa columna sedimentaria. Estudios de subsuelo han determinado espesores de hasta 500 metros para esta unidad (Gómez Omil et al., 1989).

Las muestras palinológicas fueron recolectadas por una de las autoras (Del Papa), de la sección media de la columna estratigráfica. El perfil estratigráfico con la ubicación de las muestras palinológicas se da en la Figura 3. La base de la secuencia está constituida por sedimentitas con características oxidantes (pelitas rojas) sin materia orgánica, mientras que la parte superior de la secuencia está formada por rocas con características reductoras (pelitas verdes y calizas), sin embargo, sólo se reconocieron vestigios de materia orgánica en muestra de mano y cortes petrográficos.

\section{CARACTERÍSTICAS SEDIMENTOLÓGICAS E INTERPRETACIÓN AMBIENTAL}

En el perfil de Las Tortugas se ha reconocido una serie de subambientes sedimentarios, que evolucionaron a través del tiempo y en algunos casos se asociaron lateralmente. Las características sedimentológicas en la base de la secuencia indican condiciones ambientales similares a una "llanura de fango", mientras que la sección media presenta predominio de sedimentación química (wackstones, packstones, grainstones y boudstones) con formación de una "llanura carbonática". Este subambiente se asocia hacia posiciones más profundas en la cuenca con el subambiente "lacustre 


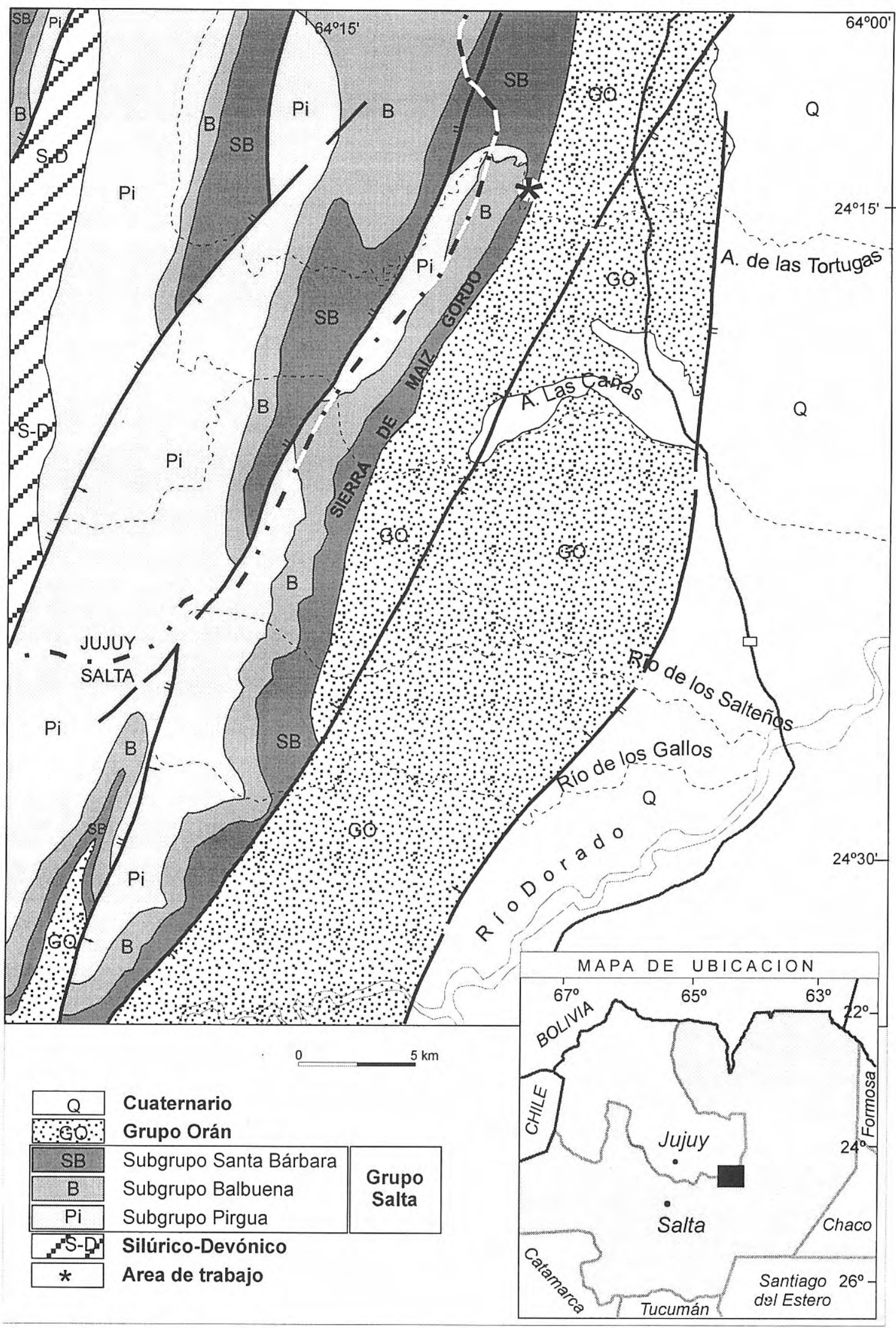

Figura 2. Mapa geológico y de ubicación.

estratificado". En la sección superior (aproximadamente $50 \mathrm{~m}$ ) la secuencia está representada por el desarrollo del subambiente "lacustre dominado por barras de oleaje"
(Fig. 3), (Del Papa, en prensa).

Los niveles palinológicamente fértiles corresponden a los subambientes "llanura carbonática "y "lacustre 


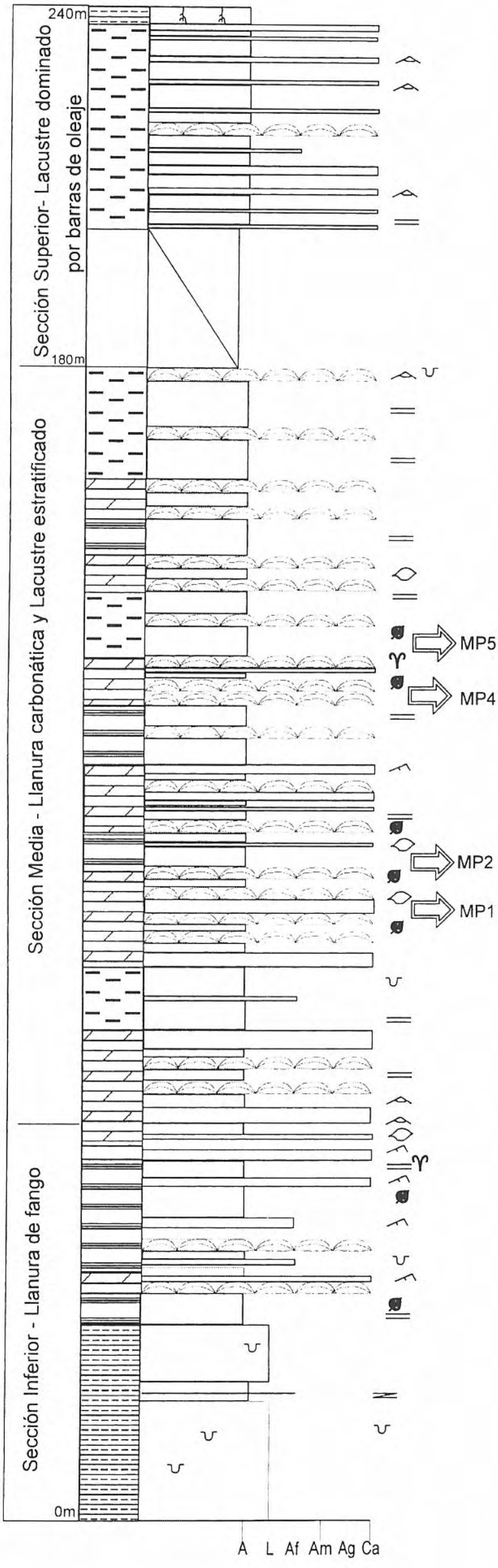

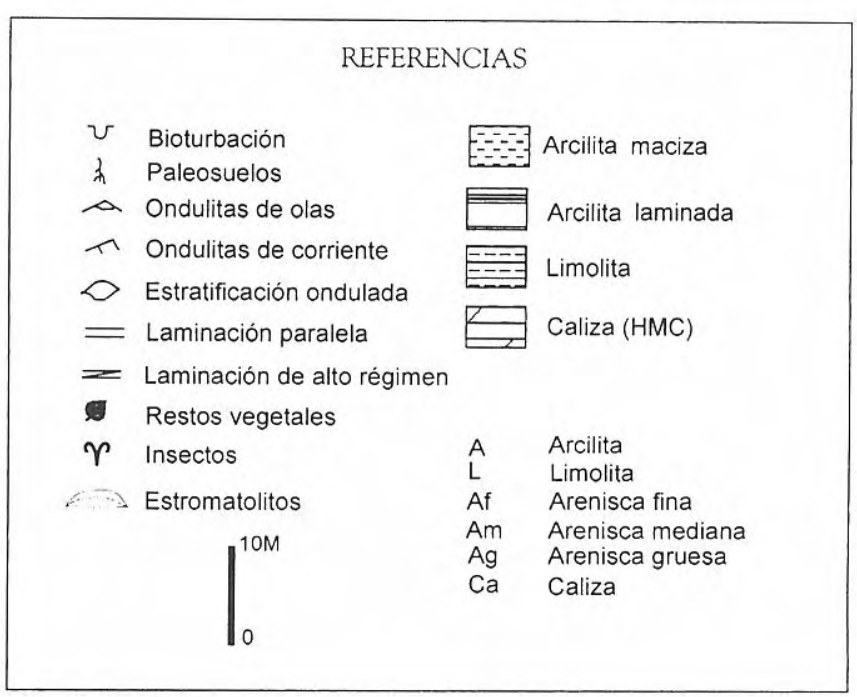

Figura 3. Perfil estratigráfico de la Secuencia Maíz Gordo en el arroyo Las Tortugas.

estratificado". El primero ha sido descripto con el nombre de "llanura de matas algáceas" por Gómez Omil et al. (1989) y está constituido principalmente por sedimentos de origen bioquímico cuya formación estuvo favorecida por la abundante proliferación de algas. Está integrado especialmente por facies de calizas boundstone, estromatolíticas y calizas laminadas; en menor proporción participan las litofacies de arcilita y limolitas verdes y calizas grainstone.

Estos depósitos formados en aguas someras reflejan un mínimo y, en ocasiones, un nulo aporte clástico, buena oxigenación de las aguas y temperaturas cálidas (Smoot, 1978; Hsü y Kelts, 1978; Castle, 1991). Temporalmente, la llanura carbonática quedaba expuesta en forma subaérea como se deduce de los numerosos biohermos que muestran evidencias de fracturación debido a procesos de meteorización.

El subambiente "lacustre estratificado" está formado por laminitas carbonáticas y arcilitas laminadas. Las laminitas se disponen rítmicamente, constituyendo niveles de hasta 3 metros de espesor y están formadas por una alternancia de láminas claras y oscuras compuestas por esparita y microesparita (HMC) y una lámina micrítica con abundantes restos de materia orgánica. Se considera que estas sedimentitas se formaron en un lago, químicamente estratificado de tipo meromíctico. Las condiciones anóxicas predominantes del fondo favorecieron la conservación de las láminas por falta de organismos bentónicos bioturbadores (Boyer, 1981).

\section{METODOLOGÍA}

El análisis paleoambiental de la Secuencia Maíz Gordo con una descripción detallada de facies sedimentarias fue realizado por Del Papa (1992) y Del Papa (en prensa).

Las muestras palinológicas fueron procesadas según las técnicas mencionadas en Volkheimer y Melendi (1976). 
Para el estudio de las palinofacies se analizaron las muestras luego de la remoción de la matriz de sedimento con los ácidos $\mathrm{HCl}$ y $\mathrm{HF}$. Se realizó un tratamiento adicional consistente de una breve oxidación. Se observó cada nivel previa y posteriormente a la oxidación con el fin de evaluar la procedencia del material amorfo. E1 material amorfo derivado de plantas terrestres tiende a ser removido más facilmente mediante la oxidación (Batten, 1981) que el material de origen algal (Batten, 1985, entre otros). Se realizaron además observaciones de fluorescencia (iluminación con luz azul o azul-UV), previa a la oxidación. Las muestran palinológicas se encuentran archivadas en el Laboratorio de Palinología de la Universidad Nacional del Sur (UNS LP). Fueron estudiadas en un microscopio Olympus B 12 (No. 2226780).

Se realizó el estudio cualitativo y cuantitativo de las asociaciones microflorísticas. Los porcentajes relativos fueron calculados sobre la suma total de 200 esporas, granos de polen y fitoplancton en 2 niveles. Los otros dos presentaron escaso material para recuento. La evaluación paleoambiental fue realizada mediante la comparación de los taxa fósiles con los taxa modernos relacionados, para lo cual se utilizó material de referencia actual y bibliografía.

El procedimiento seguido para la documentación de las palinofacies (Batten, 1987: 25) es el cálculo de porcentajes relativos mediante una valoración visual en 5 áreas del preparado, con una amplificación de x40, bajo luz transmitida. Las observaciones con microscopía de fluorescencia siguiendo a Tyson (1995: 343), se realizaron con un objetivo x 20 .

El registro de los taxa de palinomorfos, como porcentajes relativos, es documentado separadamente.

\section{CLASIFICACIÓN DE LOS CONSTITUYENTES PALINOLÓGICOS}

Según Tyson (1995), el material palinológico comprende:

Fitoclastos: Fragmentos de tejidos derivados de plantas superiores u hongos. Translúcidos u opacos. Bioestructurados o estructurados o "seudoamorfos".

Amorfo: Material no-estructurado derivado de algas nofosilizadas, o biodegradación avanzada de tejidos. Sin morfología o fábrica, a menudo con inclusiones orgánicas y pirita. Resinas.

Palinomorfos: Terrestres: esporomorfos.

Acuáticos: microplancton.

Zooclastos: Derivado de animales.

\section{PALINOESTRATIGRAFÍA}

La sección estudiada contiene especies restringidas al Paleoceno, como Retitricolporites chubutensis Arch., Verrustephanoporites simplex Leid. y Gemmatricolpites subsphaericus Arch., junto a especies del Eoceno, como Retitricolporites medius Gonz. Guzm. (Eoceno Inferior y Medio, González Guzmán, 1967).

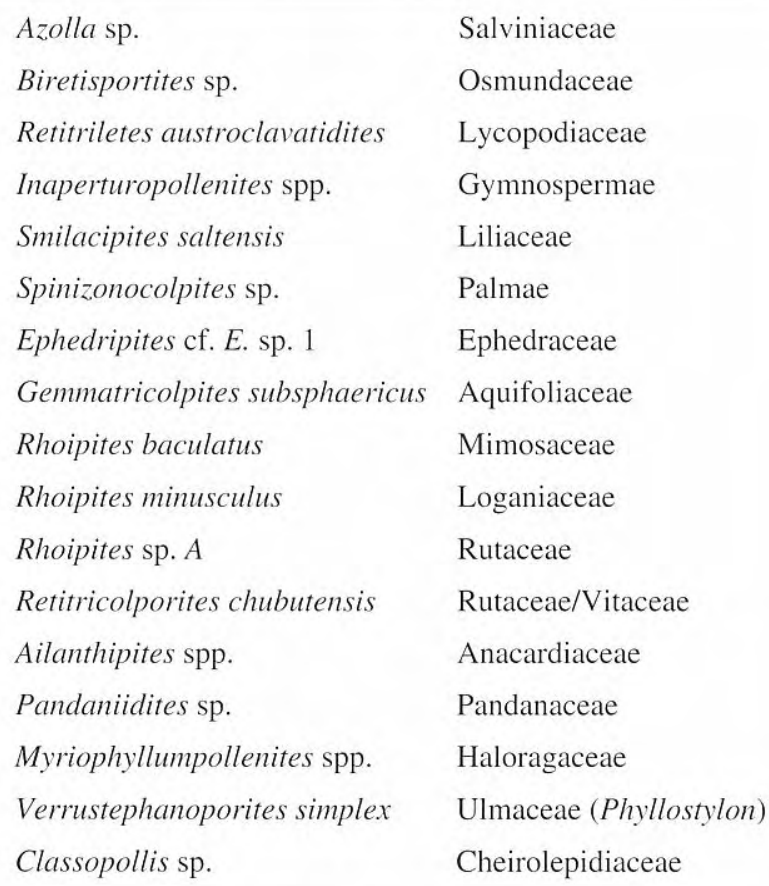

Tabla 1. Equivalentes modernos de los taxones fósiles y familias fósiles presentes en la Secuencia Maíz Gordo.

\section{PALEOCOMUNIDADES}

La asignación de los taxones fósiles (Apéndice ) a los modernos relacionados (Tabla 1) permitió caracterizar paleocomunidades para la Secuencia Maíz Gordo en la sección estudiada.

La existencia de un paleoambiente palustre estaría indicada por la presencia de Pandanaceae (Pandaniidites sp.) y Palmae (Spinizonocolpites sp.). Y lagos, por las familias Salviniaceae (Azolla sp.) y Haloragaceae (Myriophyllumpollenites spp.).

La selva subtropical húmeda está reflejada por la presencia de Ulmaceae (Verrustephanoporites simplex, hasta $23 \%$ ). Vegetación de mayor altitud estaría indicada por la presencia de las Aquifoliaceae (Gemmatricolpites subsphaericus: 4\%) Lycopodiaceae (Retitriletes austroclavatidites), Anacardiaceae (Retitricolporites spp., Ailanthipites sp.) y Rutaceae (Rhoipites sp. A) en bajos porcentajes.

Todas las muestras se caracterizan por la abundancia de polen de Ulmaceae (hasta 23\%: Verrustephanoporites simplex). La asignación probable de esta especie (Quattrocchio y Volkheimer, 1988) es a Phyllostylon, árbol dominante del actual Distrito de la Selva de Transición (350-500 m s.n.m.), dentro de la Provincia de las Yungas (Cabrera, 1976), Dominio Amazónico. Las condiciones climáticas de la Provincia de las Yungas son cálido-húmedas con lluvias principalmente estivales.

La presencia de la Familia Cheirolepidaceae (Classopollis sp.), indicadora de medios costeros y aridez, ya fue mencionada para la Formación Maíz Gordo 
en la Provincia de Jujuy (Petrulevicius, 1996). Estos hallazgos serían más jóvenes que los mencionados en la literatura para la familia Cheirolepidiaceae, por lo que no se descarta que se trate de material reelaborado.

Se registran, además, quistes de dinoflagelados nomarinos en porcentajes relativamente elevados: $25 \%$ del total de palinomorfos en la Palinofacies $\mathrm{C}$ y algo menor en la Palinofacies B (ver Palinofacies).

Siguiendo a Batten (1989), las palinofacies B y C presentan las características mencionadas para las asociaciones de quistes no marinos; o sea, el dominio de una o a lo sumo dos especies, de paredes finas y débiles, plegadas y generalmente difíciles de identificar. Se considera que la aparición de estos quistes está relacionada con un aumento de la salinidad en las masas de agua (Batten, 1989: 272).

Es de destacar la ausencia en estas asociaciones microflorísticas de Pediastrum, alga verde presente en las formaciones Tunal, Maíz Gordo (en la localidad de Corralito) y Lumbrera (Fajas Verdes I y II) (Quattrocchio y Volkheimer, 1990). Su presencia refleja condiciones estenohalinas, ricas en nutrientes y alcalinas. Tampoco ha sido registrada en la Formación Mealla (Quattrocchio et al., 1997), formada en un paleoambiente de planicie calcárea, bajo condiciones de relativa aridez.

\section{PALINOFACIES}

Según Batten (1987: 11), una palinofacies es una asociación distintiva de componentes orgánicos ácidoresistentes de una roca sedimentaria acumulada bajo determinadas condiciones y por lo tanto refleja un proceso particular o ambiente. Consideradas sin tener en cuenta el análisis de facies sedimentarias, su interpretación es inevitablemente más ambigua que cuando estos datos son combinados con el estudio sedimentológico de la roca que las contiene. El conocimiento del contexto sedimentario de una palinofacies particular es, por lo tanto, esencial para una interpretación ambiental con mayores fundamentos (Batten, 1987:12).

Se integran en este trabajo la información de facies sedimentarias y palinofacies de la sección estudiada. La información correspondiente a la frecuencia relativa de los palinomorfos calculada sobre el total de esporomorfos más fitoplancton se da en la Tabla 2. Algunos de los taxa reconocidos se presentan en la Lámina I . En la Lámina II se ilustran las palinofacies.

\section{PALINOFACIES A: PREPARADO PALINOLÓGICO} 979 - MUESTRA MP1. Lám. II, figs. 1 y 2

Fitoclastos: opacos: equidimensionales: 50\%; alargados ("blades"): $10 \%$.

Incluyen tejidos oxidados y/o carbonizados leñosos junto a partículas de carbón.

Amorfo: $40 \%$.

Palinomorfos: Escasos. Se observan sólo esporomorfos: Verrustephanoporites simplex y granos de polen tricolporados.

Observaciones: Con microscopía de fluorescencia se observa amorfo fluorescente, por lo que se presume que aunque no se observa fitoplancton, el amorfo podría derivar de la destrucción del mismo (el fitoplancton reconocido en otros niveles es de pared muy delgada). Escaso amorfo permanece después de la oxidación, por lo que provendría mayormente de plantas terrestres.

\section{FACIES SEDIMENTARIA}

Mudstone dolomicrítico laminado. Los litosomas que componen esta facies presentan geometría tabular, con espesores entre 0,60 y $1,00 \mathrm{~m}$. Está asociada mediante contacto gradacional con facies de arcilita y limolitas verdes. Está compuesta por calizas de tipo mudstone dolomítico en parejas de lámina clara y oscura de escasos milímetros de espesor. Cada lámina es granodecreciente desde microesparita a micrita. El contacto basal de la lámina clara es débilmente erosivo, y es común la presencia de materia orgánica y minerales de hierro (pirita y hematites de alteración). Las láminas oscuras, compuestas de micrita, presentan menores proporciones de estos elementos. Estas facies representan laminitas carbonáticas depositadas por debajo del nivel de base de olas, en un cuerpo de características meromícticas y constituye la facies principal del sistema deposicional lacustre estratificado.

\section{PALINOFACIES B: PREPARADO PALINOLÓGICO 980 - MUESTRA MP2. Lám. II, figs. 3-6}

Fitoclastos: opacos: equidimensionales: 70\%; alargados ("blades"): escasos.

Amorfo: 20\%. Material no estructurado pardo negruzco que incluye cuerpos globulares granulares relativamente grandes.

Palinomorfos: $10 \%$ (Esporomorfos: $85 \%$. Paleomicroplancton: $25 \%$. Calculado sobre el total de palinomorfos). Escasa diversidad de esporomorfos: 11 especies.

Observaciones: Presencia de pirita e hifas de hongos (Lám. I, Fig. 15).

Luego de la oxidación (Lám. II, fig. 5), permanece el amorfo finamente dividido de posible origen algal. Al observar con UV los preparados palinológicos no oxidados todo el amorfo fluorece.

\section{FACIES SEDIMENTARIA}

Arcilita y limolitas verdes: Esta facies es la más

Tabla 2. Distribución y porcentajes relativos de taxones selecccionados de las formaciones Tunal, Mealla, Maíz Gordo y Lumbrera. Localidades: 1. Corralito. 2. Tilián. 3. Ao. Garabatal. 4. Pampa Grande: a. Faja Verde I. b. Faja Verde II. 5. Ao. Las Tortugas. 


\begin{tabular}{|c|c|c|c|c|c|c|c|c|}
\hline TAXA & & TAL & MEALLA & & RDO & & & \\
\hline & 1 & 2 & 3 & $\overline{5}$ & 2 & $\mathbf{a}$ & $\overline{\mathbf{b}}$ & \\
\hline $\begin{array}{l}\text { Ephedripites cf. E. sp. } 1 \\
\text { Gemmatricolpites subsphaericus }\end{array}$ & $\cdots$ & $\cdots$ & $x x$ & $\cdots$ & & & & \\
\hline $\begin{array}{l}\text { Gemmatricolpites subsphaericus } \\
\text { Clavatricolpites cf. gracilis }\end{array}$ & $\mathrm{xx}$ & $\cdots$ & $\ldots$ & $\ldots$ & & & & \\
\hline Rhoipites sp. B & $\mathbf{x x}$ & $\mathrm{xx}$ & $\begin{array}{l}x x \\
\ldots\end{array}$ & $\cdots$ & & & & \\
\hline Retitricolporites sp. A & $\ldots$ & $\ldots$ & & & & & & \\
\hline Ailanthipites sp. & & $\cdots$ & & ... & & & & \\
\hline $\begin{array}{l}\text { Pandaniidites texus } \\
\text { Pandaniidites sp. }\end{array}$ & $x x$ & $\mathbf{x x}$ & $\ldots$ & $\ldots$ & & & & \\
\hline Mtchedlishvilia saltenia & $\ldots$ & $\ldots$ & & & & & & \\
\hline Myriophyllumpollenites sp. 1 & $\ldots$ & $\ldots$ & & & & & & \\
\hline Myriophyllumpollenites sp. 2 & $\cdots$ & $\ldots$ & & & & & & \\
\hline Rhoipites minusculus & $\ldots$ & $\ldots$ & $x x$ & ... & $x x$ & & & \\
\hline Rhoipites sp. A & $\ldots$ & $\ldots$ & ... & ... & oo & & & \\
\hline Verrustephanoporites simplex & oo & oo & $\mathrm{xx}$ & $x x$ & $\mathrm{xx}$ & $x x$ & $x x$ & \\
\hline Podocarpidites marwickii & $\ldots$ & $\ldots$ & & & $\ldots$ & $x x$ & $x x$ & \\
\hline Pediastrum sp. & & $x x$ & & & $\ldots$ & $\ldots$ & $\ldots$ & \\
\hline Catinipollis geiseltalensis & & & $\cdots$ & & & & & \\
\hline \begin{tabular}{|l} 
Azolla sp. \\
Biretisporites sp.
\end{tabular} & & & $\begin{array}{l}\cdots \\
\ldots\end{array}$ & $\cdots$ & & & & \\
\hline $\begin{array}{l}\text { Biretisporites sp. } \\
\text { Baculatisporites sp. }\end{array}$ & & & $\cdots$ & $\cdots$ & & & & \\
\hline Ischyosporites sp. & & & $\ldots$ & & & & & \\
\hline Apiculatisporis sp. & & & $\ldots$ & & & & & \\
\hline $\begin{array}{l}\text { Monosulcites minutiscabratus } \\
\text { Spinizonocolpites sp. }\end{array}$ & & & $\begin{array}{l}\cdots \\
\ldots\end{array}$ & & & & & \\
\hline $\begin{array}{l}\text { Spinizonocolpites sp. } \\
\text { Tricolpites communis }\end{array}$ & & & ... & $\ldots$ & & $\ldots$ & $x x$ & \\
\hline Liliacidites variegatus & & & $\ldots$ & & & & & \\
\hline Psilatricolporites salmananquensis & & & $\ldots$ & & & & & \\
\hline Rhoipites baculatus & & & ... & ... & & & & \\
\hline Rhoipites sp. & & & ... & & & & & \\
\hline $\begin{array}{l}\text { Retitricolporites chubutensis } \\
\text { Restioniidites } \text { sp. }\end{array}$ & & & $\begin{array}{l}\cdots \\
\ldots\end{array}$ & $\cdots$ & & & & \\
\hline \begin{tabular}{|l} 
Restioniidites sp. \\
Corsinipollenites menendezii
\end{tabular} & & & $\ldots$ & & $\ldots$ & $\ldots$ & $\ldots$ & \\
\hline Myriophyllumpollenites sp. & & & $\ldots$ & & & & $\cdots$ & \\
\hline Tricolpites bibaculatus & & & $\mathrm{xx}$ & & & & & \\
\hline \begin{tabular}{|l} 
T. sp. cf. $T$. reticulata \\
Tricolpites cf. reticulata
\end{tabular} & & & $\cdots$ & & & oo & oo & \\
\hline $\begin{array}{l}\text { Tricolpites cf. reticulata } \\
\text { Rousea patagonica }\end{array}$ & & & $\because \cdots$ & & & $\cdots$ & $\cdots$ & \\
\hline Psilatricolpites acerbus & & & $\begin{array}{l}x i \\
\cdots\end{array}$ & & $\ldots$ & & & \\
\hline \begin{tabular}{|l} 
Psilatricolpites inargutus \\
Psilatricolpites simplex
\end{tabular} & & & & $\ldots$ & & & & \\
\hline $\begin{array}{l}\text { Psilatricolpites simplex } \\
\text { Psilatricolpites sp. }\end{array}$ & & & $\ldots$ & & & & & \\
\hline $\begin{array}{l}\text { Psilatricolpites sp. } \\
\text { Dictyophyllidites sp. }\end{array}$ & & & & $\ldots$ & $\ldots$ & & & \\
\hline Retitricolporites medius & & & & $x x$ & $x x$ & & & \\
\hline Echistephanoporites sp. cf. E. alfonsi & & & & & $\cdots$ & & & \\
\hline Cricotriporites ef. guianensis & & & & & $\cdots$ & & & \\
\hline \begin{tabular}{|l} 
Triorites sp. \\
Cricotriporites sp. A
\end{tabular} & & & & & $\cdots$ & & & \\
\hline $\begin{array}{l}\text { Cricotriporites sp. A } \\
\text { Laevigatosporites sp. A }\end{array}$ & & & & & $\cdots$ & $\ldots$ & & \\
\hline Retitriletes austroclavatidites & & & & ... & & oo & $\ldots$ & \\
\hline Laevigatosporites sp. A & & & & & & $\ldots$ & $\ldots$ & \\
\hline \begin{tabular}{|l} 
Smilacipites saltensis \\
Smilacipites sp.
\end{tabular} & & & & ... & & $\ldots$ & $\ldots$ & \\
\hline $\begin{array}{l}\text { Smilacipites sp. } \\
\text { Gabonisporites vigourouxii }\end{array}$ & & & & & & $\cdots$ & $\cdots$ & \\
\hline $\begin{array}{l}\text { Gabonisporites vigourouxii } \\
\text { Inaperturopollenites sp. D }\end{array}$ & & & & & & $\begin{array}{l}\cdots \\
\mathbf{x x}\end{array}$ & $\begin{array}{l}\cdots \\
\mathbf{x x}\end{array}$ & \\
\hline Inaperturopollenites sp. E & & & & & & $\ldots$ & & \\
\hline $\begin{array}{l}\text { Tricolpites (Psila.) lumbrerensis } \\
\text { Tricolpites sp. A }\end{array}$ & & & & & & oo & oo & \\
\hline Nothopollenites sp. & & & & & & $\ldots$ & $\ldots$ & \\
\hline $\begin{array}{l}\text { Liquidambarpollenites cf. brandonensis } \\
\text { Inapertisporites } \mathrm{sp} . \mathrm{A}\end{array}$ & & & ... & & & oo & oo & $\ldots:$ raro \\
\hline $\begin{array}{l}\text { Inapernsportes sp. A } \\
\text { Inapertisporites ovalis }\end{array}$ & & & & & & $\cdots$ & & $(<1-5 \%)$ \\
\hline Multicellaesporites sp. & & & & & & $\cdots$ & $\cdots$ & \\
\hline Diporisporites sp. & & & & & & $\ldots$ & $\ldots$ & $x x$ : frecuente \\
\hline Diporisporites elongatus & & & & & & $\ldots$ & $\ldots$ & $(5-30 \%)$ \\
\hline Diporicellaesporites sp. & & & & & & $\ldots$ & $\ldots$ & \\
\hline $\begin{array}{l}\text { Pluricellaesporites sp. A } \\
\text { Quistes de dinoflagelados }\end{array}$ & & & & $x x$ & & $\ldots$ & $\ldots$ & $\begin{array}{c}\text { oo: abundante } \\
(>30 \%)\end{array}$ \\
\hline Acritarcos & & & & & & & & \\
\hline
\end{tabular}




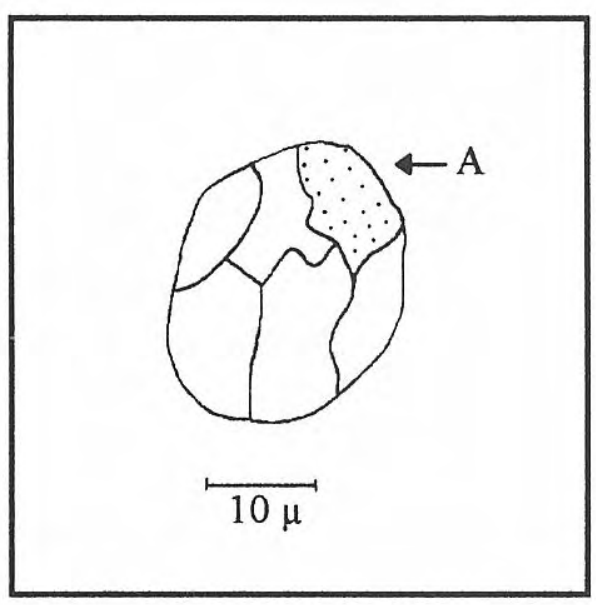

Figura 4. Quiste de dinoflagelado indet. 980a: 4,9/135. Vista ventral oblicua. (Dibujo con cámara clara). A: arqueopilo.

frecuente y característica de la Secuencia Maíz Gordo. La geometría de los niveles sedimentarios es tabular, de escasos centímetros a $3 \mathrm{~m}$ de espesor. Está constituida por arcilita y limolitas de colores verde, gris y negro. La estratificación es fina, tabular e internamente presenta laminación paralela con abundantes restos de materia orgánica.

La composición mineralógica de esta facies es variable, contiene illita, montmorillonita, analcima, dolomita-ankerita, calcita y cuarzo. Esta facies está formada por procesos de decantación, bajo condiciones tranquilas y reductoras en un cuerpo de agua estable.

\section{PALINOFACIES C: PREPARADO PALINOLÓGICO 981 - MUESTRA MP4}

Fitoclastos: opacos: 5\%. Presencia de tejidos de plantas y material leñoso.

Amorfo: $90 \%$.

Palinomorfos: 5\% (Esporomorfos: $80 \%$. Fitoplanc-ton: $20 \%$. Calculado sobre el total de palinomorfos).

Elevada diversidad de esporomorfos: 38 especies.

Observaciones: Escaso material amorfo fluorescente. Casi todo el material amorfo desaparece después de la oxidación. Por lo que se considera que el amorfo presente, previo a dicho tratamiento, provenía de plantas terrestres.

\section{FACIES SEDIMENTARIA}

Caliza laminada: Los cuerpos son de geometría tabular y cuneiforme. Esta facies está formada por láminas de wackestone-packstone y mudstone, de colores amarillento y gris. La estratificación es fina de tipos ondulada y lenticular. Las láminas $(1,5-5,0 \mathrm{~cm})$ son granodecrecientes, formadas por esparita y microesparita en la base, con frecuentes fragmentos clásticos, peloides y oolitas dispersas, mientras que el techo está compuesto por micrita y minerales de hierro. Esta facies, formada por procesos tractivo-suspensivos bajo condiciones de energía fluctuante y poca profundidad del cuerpo de agua, integra parte del subambiente de "llanura carbonática".

\section{PALINOFACIES D: PREPARADO PALINOLÓGICO 982 - MUESTRA MP5, Lám. II, figs. 7 y 8}

Fitoclastos: 5\%.

Amorfo: 90\% .

Palinomorfos: 5\% (Sólo esporomorfos. Escasa diversidad: 4 especies).

Observaciones: Aproximadamente la mitad del amorfo fluorece.

\section{FACIES SEDIMENTARIA}

Arcilita y limolitas verdes: Constituida por arcilita y, en menor proporción, niveles de limolitas, en estratos finos, tabulares. La característica principal de este nivel es la ausencia de laminación, se presenta maciza y con mayor espesor (5 metros) que en la Palinofacies B.

\section{COMPARACIONES CON REGISTROS PREVIOS}

La Secuencia Maíz Gordo fue previamente estudiada palinológicamente (Volkheimer et al., 1984) en la Quebrada de Corralito, Valle de Lerma (Subcuenca de

\section{Lámina I}

1 Biretisporites sp. 981b: 5,5/130,5. Vista proximal.

2-3 Spinizonocolpites sp. 2. $981 \mathrm{~b}: 7,2 / 128,4$. Vista ecuatorial. 3. 980a: 5,6/133,9. Vista ? polar.

4 Psilatricolpites inargutus (Mc Intyre) Archangelsky 1973. 981a: 17,9/143,9. Vista polar.

5 Smilacipites saltensis Quattrocchio 1978 a. $981 \mathrm{~b}$ : 9,5/131,6.

6 Psilatricolpites sp. 981a: 2,6/147,5. Vista ecuatorial.

7 Rhoipites minusculus Archangelsky 1973. 981a: 23/144,9. Vista ecuatorial.

8 Striatricolporites sp. 918b: 6,4/140. Vista ecuatorial oblicua.
9 Retitricolporites medius González Guzmán 1967.

10 Pandaniidites sp. 981a: 17,9/141,2. Vista ecuatorial.

11 Classopollis sp. 981a: 21,9/141. Vista polar.

12 Verrustephanoporites simplex Leidelmeyer 1966. 981b: $9,5 / 128,2$. Vista polar.

13 Quiste de dinoflagelado indet. 980 a: 4,9/135.

14 Leiosphaeridia sp. 981a: 19,8/146.

15 Quiste de dinoflagelado indet. 981b: 9,8/146. Vista lateral.

16 ?Acritarca indeterminada. $981 \mathrm{~b}: 7 / 128$.

17 Hifas de hongos. 980a: 13,9/135,5. 
Lámina I

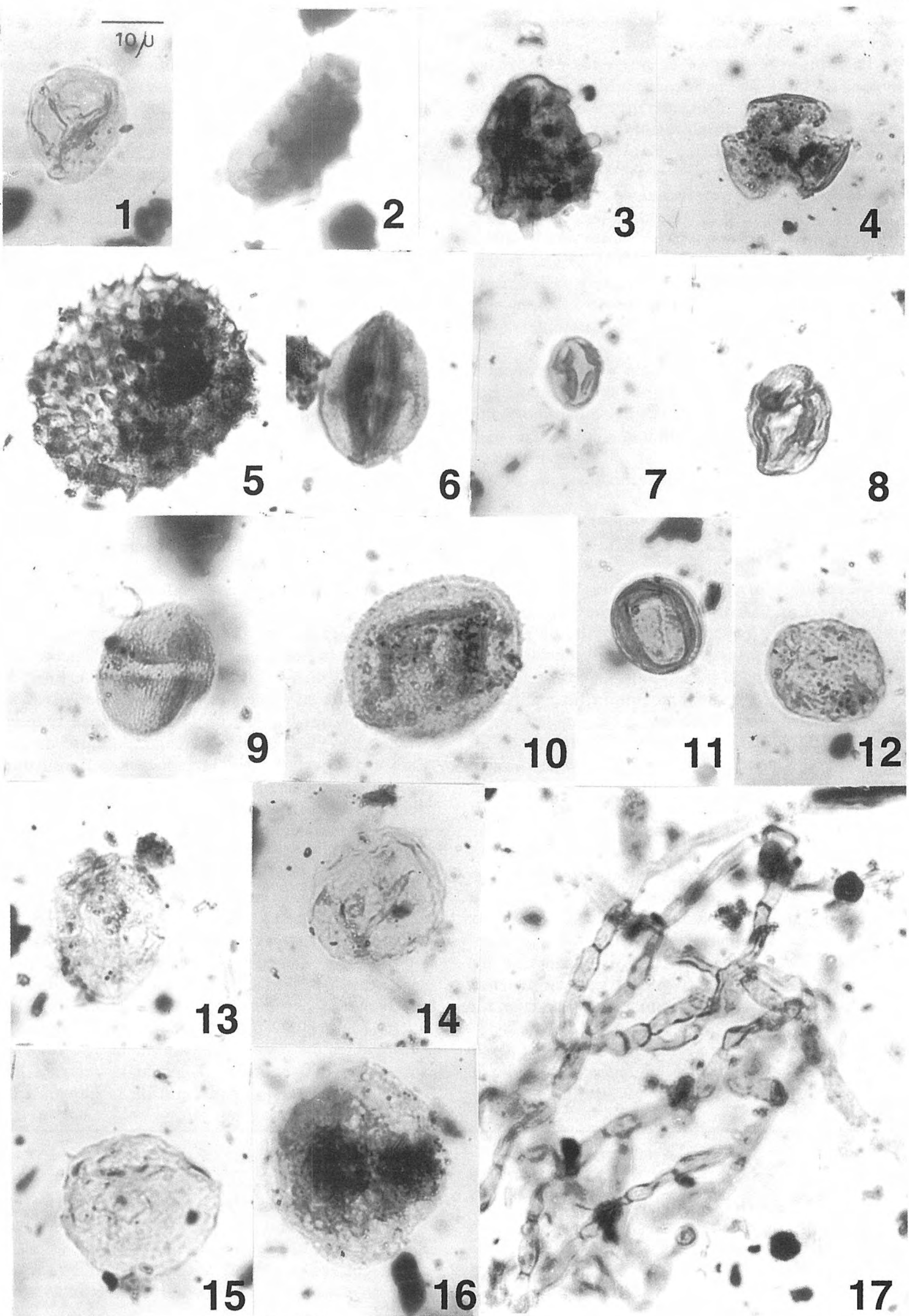

Revista Española de Paleontología, 15 (1), 2000. 
Alemanía). El único nivel fértil corresponde al tercio superior de la unidad en facies pelíticas.

De la comparación con el presente estudio se desprende (Tabla 2): que la Secuencia Maíz Gordo en la Quebrada de Corralito presenta una mayor abundancia de Rhoipites sp. A (Rutaceae) indicando la existencia de una paleocomunidad de altura (montana) y relativa aridez. Otros elementos montanos son las Podocarpaceae, Gunneraceae y Hamamelidaceae. Presencia de Pediastrum y ausencia de fitoplancton (quistes de dinoflagelados y acritarcos) indicando condiciones dulceacuícolas para el nivel considerado. Las secciones estratigráficas en la Quebrada de Corralito y en el Arroyo Las Tortugas (éste trabajo), representarían subambientes distintos y posiciones paleogeográficas diferentes en la Cuenca del Grupo Salta.

$\mathrm{Si}$ se compara los taxa presentes y sus frecuencias relativas en la Secuencia Maíz Gordo en el Arroyo Las Tortugas con las otras Formaciones del Subgrupo Santa Bárbara (Tabla 2), se observa similitud con la "Faja Gris" de la Formación Mealla, la que se interpreta depositada en un ambiente palustre a lacustre (Quattrocchio et al., 1997).

\section{CONCLUSIONES}

La Secuencia Maíz Gordo (Sección Media) en la localidad de Las Tortugas está caracterizada por sedimentación pelitíco-carbonática ocurrida en una cuenca lacustre. El principal factor de control de las facies estuvo determinado por el quimismo del agua y la escasa profundidad, siendo frecuente la formación de ciclos de inundación-somerización.

La facies de mudstone dolomicrítico laminado es interpretada como laminitas carbonáticas depositadas por debajo de la acción de las olas. La conservación de la laminación paralela sustenta la idea de un fondo anóxico con escasa actividad orgánica. La Palinofacies A, reconocida en ella, presenta sólo esporomorfos, fitoclastos opacos dominantes y material amorfo proveniente mayormente de plantas terrestres, aunque se observa con microcopia de fluorescencia escaso amorfo proveniente de destrucción de fitoplancton.

La facies de arcilita y limolitas verdes se ha interpretado como producto de decantación de material en suspensión bajo condiciones reductoras en un lago. La Palinofacies B reconocida presenta dominio de fitoclastos opacos, el amorfo en parte corresponde a material algal. Se presentan quistes de dinoflagelados, que indican condiciones de somerización y relativa salinidad, pirita (condiciones reductoras) y hongos (hifas) responsables de la biodegradación. En la Palinofacies D, también dentro de la facies de arcilita y limolitas verdes, domina el amorfo parcialmente algal con escasos fitoclastos y baja diversidad de esporomorfos. El fitoplancton habría estado presente, pero sólo estaría representado por el amorfo fluorescente. La diferencia en ambas palinofacies radicaría en que en la facies de arcilita y limolitas verdes en MP5 (Palinofacies D) es de mayor espesor, homogénea y masiva y en la Palinofacies B, presenta laminación paralela muy marcada. La cantidad presente de amorfo es indicativa de conservación en condiciones anóxicas pero con una degradación inicial, en particular de hongos, bacterias e invertebrados, que habría tenido lugar en condiciones aeróbicas, acuáticas y aún parcialmente en ambientes terrestres. Se ha observado materia amorfa membranosa, esponjosa hasta granular en los niveles estudiados, característica de ambientes nomarinos (Batten, 1983).

La facies de caliza laminada representa medios de energía fluctuante de moderada a baja, con mecanismos de tipo tractivo-suspensivo y sedimentación química predominante. La presencia de estructuras debidas a oleaje sugiere condiciones muy someras. La Palinofacies $\mathrm{C}$ reconocida en ella presenta dominancia de material amorfo; la presencia de quistes de dinoflagelados estaría relacionada con un aumento en la salinidad de la masa de agua.

Los porcentajes de Ulmaceae son menores con respecto a la Formación Tunal, unidad lateral de la F. Olmedo (Daniense) (Quattrocchio y Volkheimer, 1988). Su presencia en porcentajes menores podría indicar una retracción del bosque de Ulmaceae con respecto al Daniense o una posición cercana al centro de cuenca. Esto último estaría evidenciado por la dominancia de fitoclastos opacos en las palinofacies A y B. El cociente de resistencia del material opaco con respecto al translúcido aumenta en relación con la distancia del área de aporte por degradación selectiva o dispersión selectiva.

La unidad del Grupo Salta más afín a la Secuencia Maíz Gordo sería la "Faja Gris" de la Formación Mealla (Quattrocchio et al., 1997) que presenta un contenido palinológico y características sedimentológicas similares.

El estudio palinológico de la Formación Maíz Gordo en Quebrada Corralito (Volkheimer et al., 1984) refleja un paleoambiente de sedimentación diferente, bajo condiciones de agua dulce (presencia de Pediastrum y ausencia de quistes de dinoflagelados) y cercanía de áreas elevadas (borde sudoccidental de la cuenca), por la

\section{Lámina II}

1-3 Preparados después del tratamiento con HCL y HF. 1. Muestra 979. Amorfo (finamente dividido) y fitoclastos angulosos. x400. 2. Muestra 979. Amorfo esponjoso. x 1000. 3. Muestra 980. Amorfo finamente dividido y fitoclastos equidimensionales. $\mathrm{x} 400$.
4-8 Preparados después de leve oxidación. 4. Muestra 980. Amorfo granular. x1000 5. Muestra 980. Amorfo finamente dividido. x 400. 6. Muestra 980. Fitoclasto degradado. x 1000. 7. Muestra 982. Amorfo abundante con escasos fitoclastos. x 400. 8. Muestra 982. Amorfo fibroso x 1000 . 


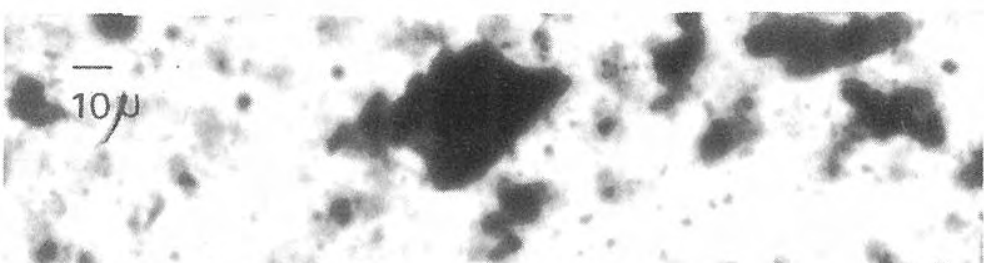
an a sine

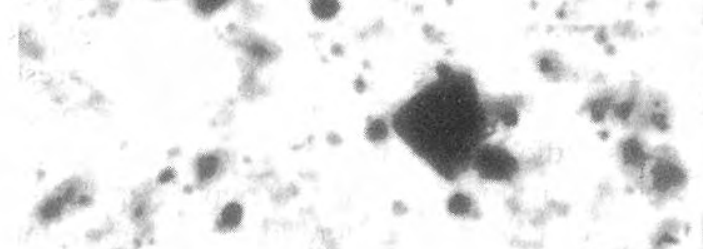

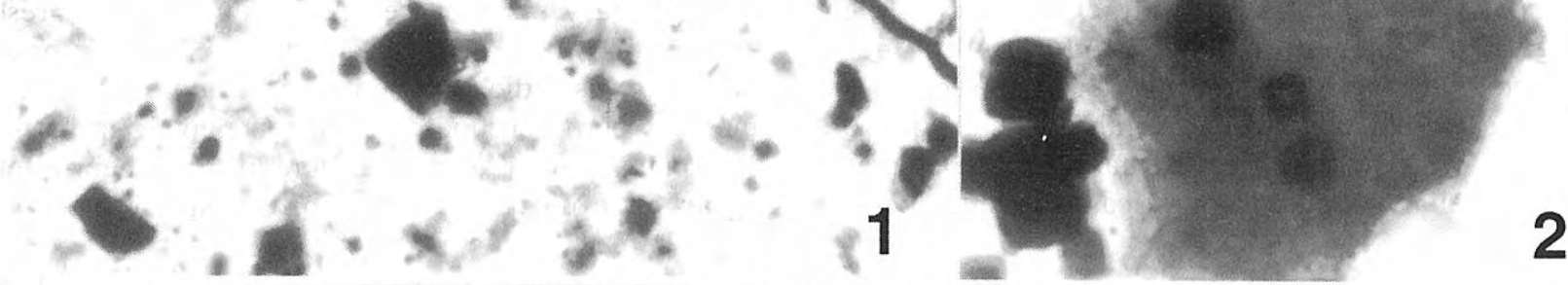

. . + +...

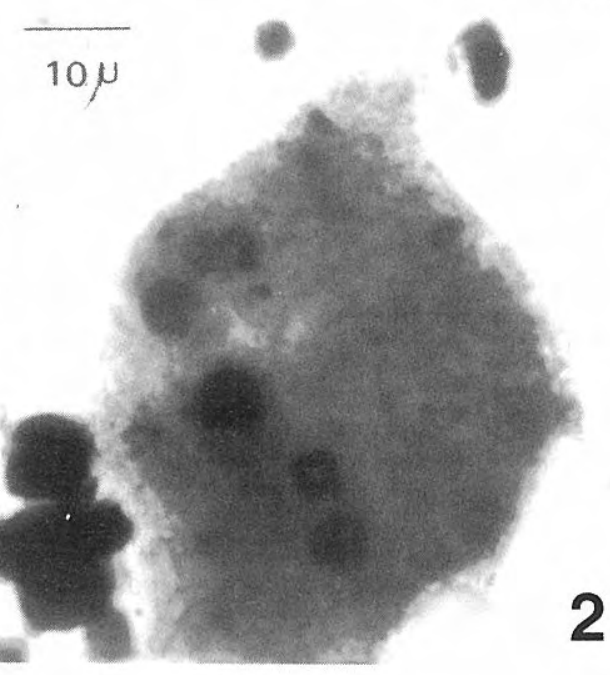

$\therefore=$

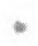

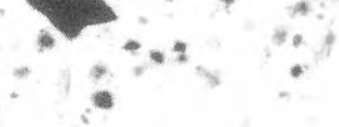

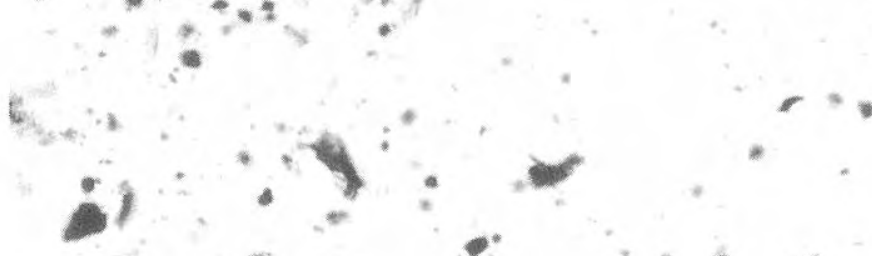

$\because+\ldots+\ldots$

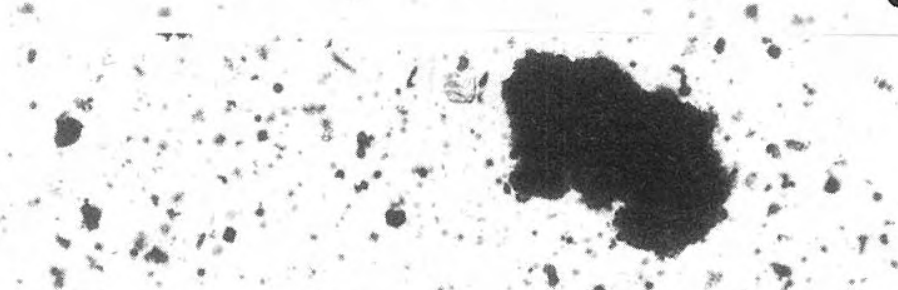

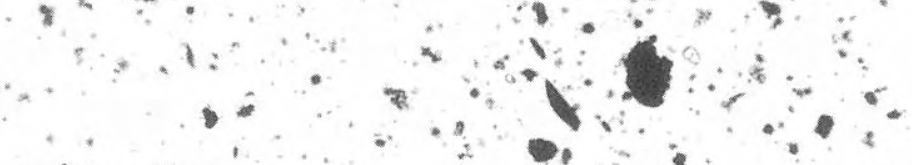

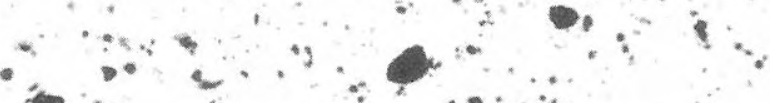

2

4

$\therefore \quad \because 0^{2}+5$

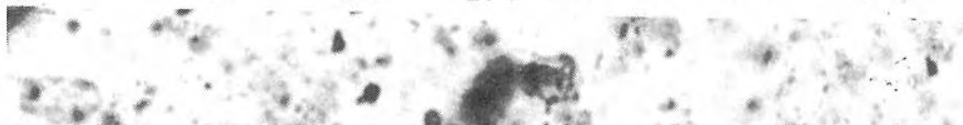

205.

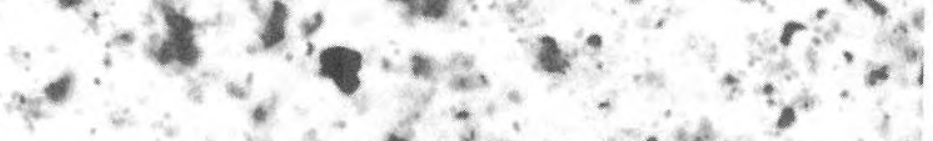

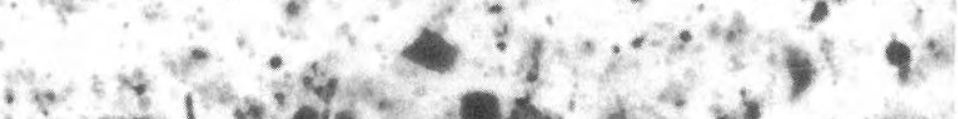

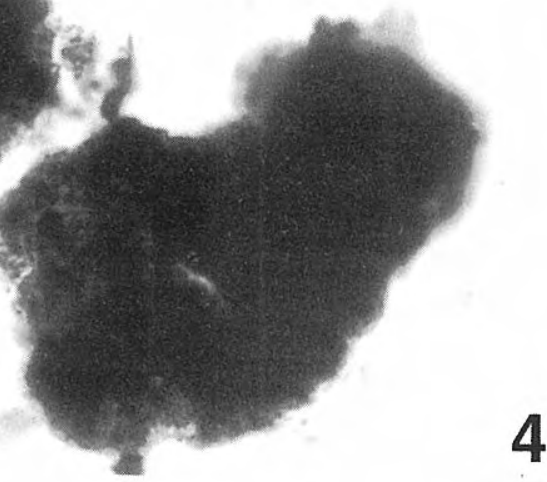
b. at $=0.78$ 
presencia de mayor diversidad y abundancia de especies relacionadas actualmente con ambiente montano.

\section{AGRADECIMIENTOS}

Las tareas de campaña fueron financiadas por el Consejo de Investigación, Universidad Nacional de Salta, a través de los Proyectos $N^{\circ} 345$ y $N^{\circ}$ 626. Se agradece a la Dra. Maquillas y al Prof. Batten la lectura crítica del manuscrito y las sugerencias realizadas. Al Prof. Batten y al Dr. Tyson los aportes referentes al análisis palinofacial. A la Secretaría Nacional de Ciencia y Técnica, al CONICET y a la Universidad Nacional del Sur, el apoyo económico.

\section{BIBLIOGRAFÍA}

Archangelsky, S. 1973. Palinología del Paleoceno de Chubut. I Descripciones Sistemáticas. Ameghiniana, 10, 339-399.

Batten, D.J. 1981. Palynofacies, organic maturation and source potential for petroleum. Organic maturation studies and fossil fuel exploration (Ed. J. Brooks). Academic Press, London, 201-223.

Batten, D.J. 1983. Identification of amorphous sedimentary organic matter by transmitted light microscopy. In: Petroleum geochemistry and exploration of Europe (Ed. J. Brooks ). Geological Society, London, Special Publication, 11, 275-287.

Batten, D.J. 1985. Coccolith moulds in sedimentary organic matter and their use in palynofacies analysis. Journal of Micropalaeontology, 4, 111-116.

Batten, D.J. 1987. Application of palynofacies analysis to interpretation of palaeoenvironments and source potential for hydrocarbons. Course notes. $34 \mathrm{pp}$. (Unpublished).

Batten, D.J. 1989. Cretaceous freshwater dinoflagellates. Short Communication. Cretaceous Research, 10, 271273.

Boyer, R. 1981. Tertiary lacustrine sediments from Sentinel Butte, North Dakota and the sedimentary record of ectongenic meromixis. Journal of Sedimentary Petrology, 51, 429-440.

Cabrera, A. 1976. Regiones Fitogeográficas Argentinas. Enciclopedia Argentina de Agricultura y Jardinería, 2 (1), 1-85.

Castle, J, 1991. Sedimentation in Eocene Lake Uinta (Lower Green River Formation), Nothwestern Uinta Basin, Utah. In: Lacustrine Basin Exploration-Case Studies and Modern Analogs (Ed. B. J. Katz). American Association Petroleum Geologist, Memoir 50, 243-264.

Del Papa, C.E., 1992. Ciclos de somerización en la secuencia Maíz Gordo (Terciario Inferior), noroeste argentino. Cuarta Reunión Argentina de Sedimentología, 1, 41-45. La Plata

Del Papa, C.E., 1994. Estratigrafía y Sedimentología de la Formación Maíz Gordo (Paleoceno) en el sur de la cuenca del Grupo Salta. Tesis Doctoral, Universidad Nacional de Salta, 124 pp. (inédita).
Del Papa, C.E., (en prensa). Sedimentation on a ramp type lake margin: Paleocene-Eocene Maiz Gordo Formation, Northwestern Argentina. Journal of South American Earth Sciences.

Galliski, M.A. and Viramonte, J.G., 1988. The Cretaceous paleorift in Northwestern Argentina: a petrologic approach. Journal of South American Earth Sciences, 1, 329-242.

Gómez Omil, R.J., Boll, A. y Hernández, R.M., 1989. Cuenca cretácico-terciaria del Noroeste argentino (Grupo Salta). In: Cuencas Sedimentarias Argentinas (Eds. G.A. Chebli y L.A. Spalletti). Universidad Nacional de Tucumán, Serie de Correlación Geológica, 6, 43-64.

González Guzmán, A.E. 1967. A Palynological study on the Upper Los Cuervos and Mirador Formations. (Lower and Middle Eocene; Tibú Area, Colombia). Leiden, 1-67.

Hsü, K.J. and Kelts, K. 1978. Late Neogene sedimentation in the Bláck Sea. In: Modern and Ancient Lake Sediments (Eds. A. Matter and M.E. Tucker). International Association Sedimentologist, Special Publication, 2, 7-41.

Krutzch, W. 1963. Atlas der Mittel-und Juntertiären Dispersen Sporen und Polleen sowie der Mikroplanktonformen des Nördlichen Mitteleuropas, II: Die Sporen der Anthocerotaceae una der Lycopodiaceae. Veb Gustav Fischer Verlag Jena, Berlin, $141 \mathrm{pp}$.

Leidelmeyer, P. 1966. The Paleocene and Lower Eocene pollen flora of Guyana. Leidse geologische Mededelingen, 38, 49-70.

Moreno, J., 1970. Estratigrafía y paleogeografía del Cretácico Superior en la cuenca del norte argentino, con especial mención de los Subgrupos Balbuena y Sánta Barbara. Revista de la Asociación Geológica Argentina, 25 (1), 9-44.

Petrulevicius, J. 1996. Una nueva localidad fosilífera del Paleoceno Superior (Formación Maíz Gordo) en el Noroeste Argentino. Congreso del Paleógeno de América del Sur (La Pampa, 1996). Resúmenes, 20.

Quattrocchio, M.1978a. Datos paleoecológicos y paleoclimatológicos de la Formación Lumbrera (Grupo Salta). Ameghiniana, 15, 173-181.

Quattrocchio, M. 1978b Contribución al conocimiento de la Palinología Estratigráfica de la Formación Lumbrera (Terciario inferior, Grupo Salta). Ameghiniana, 15, 285300.

Quattrocchio, M. 1980. Estudio palinológico preliminar de la Formación Lumbrera (Grupo Salta), loc. Pampa Grande, provincia de Salta. II Congreso Argentino de Paleontología y Bioestratigrafía y I Congreso Latinoamericano de Paleontología, Actas, 2, 131-149.

Quattrocchio, M. y Volkheimer, W. 1988. Microfloras de los estratos limítrofes entre Cretácico y Terciario en las localidades de Tilián y Corralito, Cuenca de Salta. Descripciones Sistemáticas. IV Congreso Argentino de Paleontología y Bioestratigrafía, Actas, 3, 109-120. Mendoza, 1986.

Quattrocchio, M. and Volkheimer, W. 1990. Paleogene paleoenvironmental trends as reflected by palynological assemblage types, Salta Basin, NW Argentina. Neues. 
Jahrbuch für Geologie und Paläontologie. Abhandlung, 181, 377-396.

Quattrocchiio, M. and Volkheimer, W. (en prensa). Paleoclimatic changes during the Paleocene-Lower Eocene, in Salta Basin, NW of Argentina. In: Southern Hemisphere Paleo- and Neoclimates. Methods, Concepts and Problems (Eds. W. Volkheimer and J. Smolka). Cambridge University Press.

Quattrocchio, M., Marquillas, R. y Volkheimer, W. 1988. Palinología, paleoambientes y edad de la Formación Tunal, Cuenca del Grupo Salta (Cretácio-Eoceno), República Argentina. IV Congreso Argentino de Paleontología y Bioestratigrafía, Actas, 3, 95-107.

Quattrocchio, M., Volkheimer, W. and Del Papa, C. E. 1997. Palynology and Paleoenvironment of "Faja Gris", Mealla Formation (Salta Group) at Garabatal Creek (NW Argentina). Palynology, 21, 231-247.

Salfity, J.A., 1980. Estratigrafía de la Formación Lecho (Cretácico) en la cuenca andina del Norte Argentino. Tesis Doctoral, Universidad Nacional de Salta, 91 pp. (inédita).

Salfity, J.A. 1982. Evolución paleogeográfica del Grupo Salta (Cretácico-Eogénico), Argentina. V Congreso Latinoamericano de Geología, 1, 11-26, Buenos Aires.

Smoot, J.P. 1978. Origin of carbonate sediments in the Wilkins Peaks Meamber of lacustrine Green River
Formation (Eocene), Wyoming, U.S.A. In: Modern and Ancient Lake Sediments (Eds. A. Matter and M.E. Tucker). International Association Sedimentologist, Special Publication, 2, 215-240.

Turner, J.C. 1959. Estratigrafía del cordón de Escaya y de la sierra Rinconada. Revista de la Asociación Geológica Argentina, 15 (1), 15-39.

Tyson, R.V. 1995. Sedimentary Organic Matter. Organic facies and palynofacies. Chapman \& Hall Eds., 615 pp. London.

Vail, P., Mitchum, R., Todd, R., Widmier, J.G., Thompson, J.M., Sagree, S., Bubb, J. and Hatelid, W. 1977. Seismic stratigraphy and global changes of sea level. In: Seismic Stratigraphy-Applications to Hydrocarbon Exploration (Ed. C.E. Clayton). American Association Petroleum Geologist, Memoir 26, 49-212.

Volkheimer, W. y Melendi, D. 1976. Palinomorfos como fósiles guía (3ra. Parte). Técnicas de laboratorio palinológico. Revista Minera, Geología y Mineralogía. Sociedad Argentina de Minería y Geología, 34 (1/2), 19 30, Buenos Aires.

Volkheimer, W., Quattrocchio, M. y Salfity, J., 1984. Datos palinológicos de la Formación Maíz Gordo, Terciario inferior de la Cuenca de Salta. IX Congreso Geológico Argentino, Actas, 4, 523-538. Bariloche.
Manuscrito recibido: 17 de mayo, 1999 Manuscrito aceptado: 22 de septiembre, 1999

\section{APÉNDICE}

\section{LISTA TAXONÓMICA}

\section{Laevigati}

Azolla sp.

Biretisportites sp. (en Quattrocchio, 1980)

\section{Murornati}

Retitriletes austroclavatidites (Cook.) Döring, Krutzsch, Mai y Schultz (en Krutzsch, 1963)

\author{
Aletes \\ Inaperturopollenites spp. \\ Smilacipites saltensis Quattrocchio 1978a
}

\section{Plicates}

Spinizonocolpites sp. (en Archangelsky, 1973)

Ephedripites cf. E. sp. 1 Frederiksen et al. 1983 (en Quattrocchio y Volkheimer, 1988)

Gemmatricolpites subsphaericus Archangelsky 1973

Clavatricolpites cf. gracilis González Guzmán 1967

Tricolpites communis Archangelsky 1973

Psilatricolpites inargutus (Mc Intyre) Archangelsky 1973

Psilatricolpites sp. ( en Archangelsky, 1973)

Rhoipites baculatus Archangelsky 1973
Rhoipites minusculus Archangelsky 1973

Rhoipites sp. A (en Quattrocchio, 1978a)

Retitricolporites chubutensis Archangelsky 1973

Retitricolporites medius González Guzmán 1967

Ailanthipites spp.

Striatricolporites spp.

\section{Poroses}

Pandaniidites sp. (en Archangelsky, 1973)

Myriophyllumpollenites spp.

Verrustephanoporites simplex Leidelmeyer 1966

Classopollis sp.

\section{Fitoplancton}

¿Leiosphaeridia spp.

Quiste de dinoflagelado indeterminado

\section{SISTEMÁTICA}

Quiste de dinoflagelado indet.

Fig. 4; Lám. I, figs. 13 y 15

Descripción: Quistes de dinoflagelados proximales, pequeños, de pared delgada y contorno subcircular. 
Autofragma solamente, muy plegado. Paratabulación no discernible, y sólo la indicación de paratabulación está expresada por el arqueopilo apical y suturas accesorias al arqueopilo sugiriendo la presencia de 4 paraplacas apicales y 6 paraplacas precingulares. Presencia de algunas crestas bajas. Opérculo libre. Presencia de la hendidura sulcal ("sulcal notch") formada cuando Iu se extiende hacia el paracíngulo para encontrar la paraplaca ai. La forma del arqueopilo indica que es de tipo (4A).
Dimensiones: Diámetro ecuatorial máximo: 20-30 $\mu \mathrm{m}$ (20 ejemplares).

Material ilustrado: 980 a: 4,9/135; 981b: 9,7/146.

Observaciones: ?Leiosphaeridia spp. mencionadas en este trabajo poseen la misma descripción pero posiblemente por poseer el opérculo adherido no se pudo determinar su afinidad con los quistes de dinoflagelados. 